\title{
Cytogenetical Studies in Rhoeo spathacea (Commelinaceae) II. Characterization of an acrotrisomic plant
}

\author{
Armando Garcia-Velazquez \\ C.P. 56230, Montecillo, Mex. Mexico
}

Accepted July 17, 1995

Structural hybridity for translocations, or segmental interchanges, has played a more important role in plant evolution than other chromosomal aberrations. The classic group of plants for studies of hybridity for segmental interchanges is Oenothera. They regularly form rings rather than pairs of chromosomes at meiosis. The chromosome rings are nearly always oriented on the meiotic metaphase spindle in a particular way so that alternate chromosomes go to the same pole. In this way such distribution of chromosomes yields two supergenes. From the extensive work on Oenothera the fullest understanding of the origin and mechanism of the system has been derived. It has been assumed that other permanent hybrids arose as in Oenothera, i.e., by accumulation of interchanges new cases appear to be more complex. Goldblatt (1980) found in Homeria three cytotypes differing by Robertsonian fusion, and show complete rings at meiosis. However, in the majority of cases, it has not been possible to establish the nature and size of the interchanges: there is some debate on the exact mode of origin of the system: in Oenothera Cleland (1972) proposes whole arm interchanges, whereas Darlington (1929) favors segmental interchanges. Segmental interchanges has been found in several Commelinaceae (Lin and Paddock 1973, Kenton et al. 1987). Permanent hybridity in Gibasis pulchella, another Commelinaceae, has allowed the identification of segmental interchanges confined to terminal regions and of unequal size; this adds new dimension to the study of this phenomenon (Kenton et al. 1987).

There are many studies on meiosis in Rhoeo. Spontaneous triploids (Desai 1965, Lin et al. 1984) and tetraploids (Walters and Gerstel 1948, Garcia 1991) have been obtained in Rhoeo spathacea. Two exceptional mutants were found which exhibits up to 12 univalents at MI and also produce unreduced gametes by failure of second cytokinesis. These mutants were identified as desynaptic-SDR (García 1991, 1994).

Natarajan and Natarajan (1972) found that the karyotype of $R$ hoeo includes 6 metacentric and 6 submetacentric chromosomes. However, until now there is no any proposal of a standard karyotype in Rhoeo.

This paper describes the somatic karyotype analysis of an acrotrisomic plant derived from a desynaptic-SDR mutant (García 1991). The meiotic behavior and pollen cytology are also described. This plant is the first aneuploid ever observed in Rhoeo spathacea.

\section{Material and methods}

The material used in this study were a desynaptic-SDR mutant, GAVA 1.1. and a spontaneous trisomic plant GAVA 1.1.15 obtained by selfing the mutant. All plants growing in a greenhouse in Chapingo, Mex.

For mitotic karyotype analysis, root tips were collected in the morning and pretreated in an aqueous solution $0.002 \mathrm{M}$ of 8 -hydroxyquinoline at $14^{\circ} \mathrm{C}$ for up to $6 \mathrm{hr}$. Then they were fixed in a fresh mixture of ethanol and glacial acetic acid $(3: 1 \mathrm{v} / \mathrm{v})$, and kept in the fixative overnight. After $8 \mathrm{~min}$ hydrolysis in $1 \mathrm{~N} \mathrm{HCl}$ at $60^{\circ} \mathrm{C}$ Feulgen staining was used for $5 \mathrm{~min}$ at 
$60^{\circ} \mathrm{C}$. Maceration in cytase (gut juice from the snail, Helix sp.) for $2 \mathrm{hr}$ at room temperature and then root tips were squashed in $45 \%$ acetic acid (García 1991). Ten cells were drawn with a Zeiss tube attachment at $\times 2000$ magnification and photography performed with Kodalith film using a FOMI III microscope. Metaphase chromosomes were identified by using two variables simultaneously: relative length $(\mathrm{L} \%)$ and arm ratio $(r)$ as proposed by Tjio and Hagberg (1951). The karyotype of the maternal plant was used as a control. Cytological data on chromosome behavior at meiosis were obtained from anthers of suitable size squashed directly into $1.8 \%$ propionic orcein.

\section{Results and discussion}

Karyotype analysis

Fig. 1 shows somatic complements of parental diploid (GAVA 1.1) and acrotrisomic (GAVA 1.1.15) plants. Minor variations in relative length and arm ratio from one cell to another were common. Therefore, it was considered more appropriate to use the method

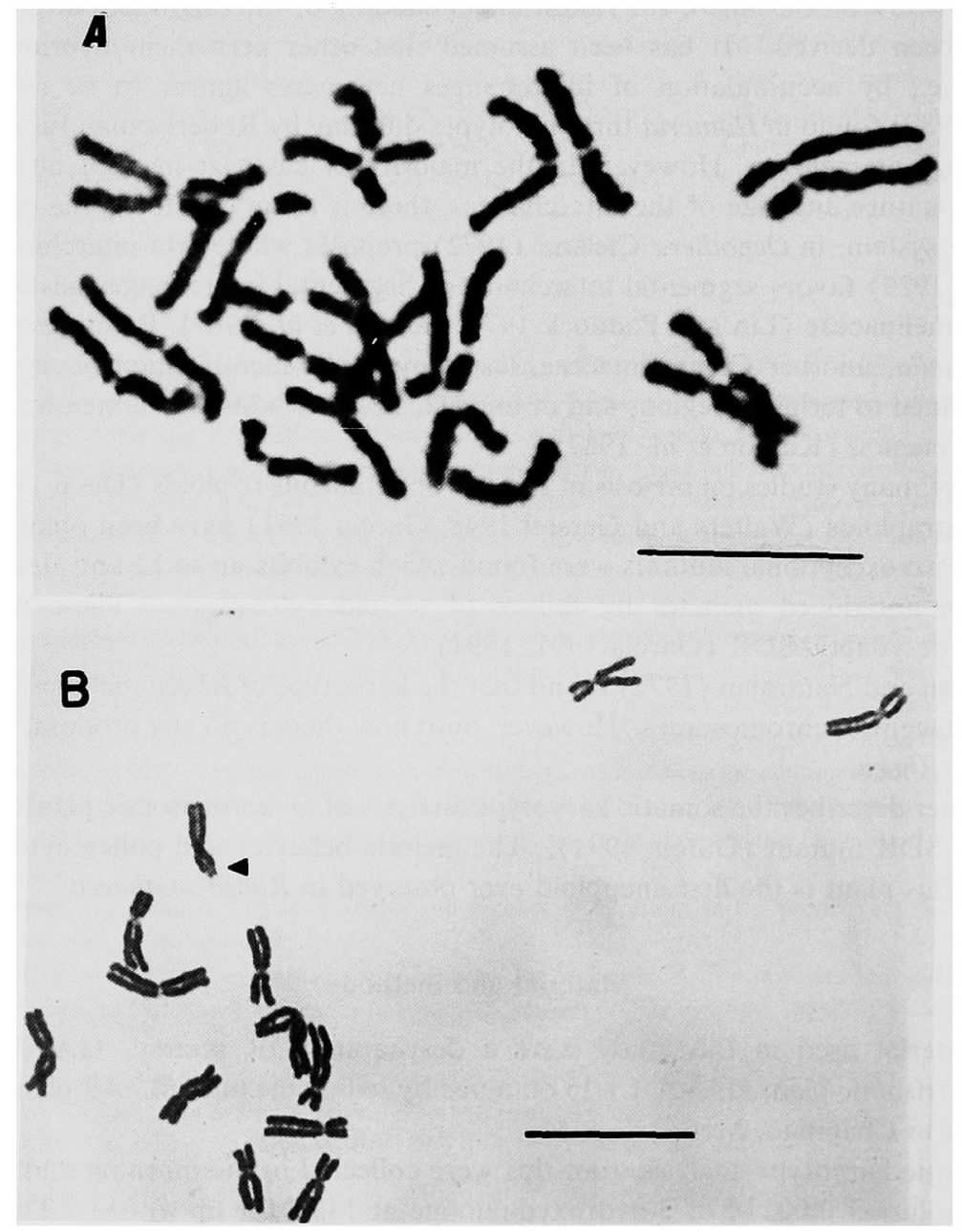

Fig. 1. Somatic cells of Rhoeo spathacea. A. disomic plant GAVA $1.1(2 n=12)$. B. Trisomic plant GAVA 1.1.15 $(2 n=13)$, acrocentric chromosome (arrow). Bar $=10 \mu \mathrm{m}$. 
proposed by Tjio and Hagberg (1951) based on relative length data on the X-axis; rather than using a statistical quantitative analysis. The average of relative length (L\%) and arm ratio (r) are listed in Table 1 and displayed in Fig. 2 for all the chromosomes in both plants. There is no indication of the nature of the extra chromosome (number 7 in Table 1). It appeared as an acrocentric with a relative length of $7.6 \%$ and an arm ratio of 12.0 . However, based on the length of the long arm, the acrocentric can be compared with the longest arm of chromosome 1.

From these comparative analyses of karyotype of the plant GAVA 1.1 and its selfed plant GAVA 1.1.15, it is clear (Fig. 2) that some unexpected changes have occurred. Chromosomes $2,3,4,5$ and 7 changed markedly in both arm ratio (r) and relative length (L\%), from one generation to the next. These changes suggest that, in meiosis in the plant GAVA 1.1, unequal interchanges occur in long and short arms, mostly in the largest chromosomes. There are many changes in both relative length ( $\mathrm{L} \%)$ and arm ratio $(\mathrm{r})$ of the chromosomes from the plant GAVA 1.1 to the plant GAVA 1.1.15 to assume a random occurrence. It seems to be a result

Table 1. Relative length ( $L \%)$ andharm ratio (r) of chromosomes in trisomic and disomic Rhoeo spathacea

\begin{tabular}{ccccc}
\hline \multirow{2}{*}{$\begin{array}{c}\text { Chromosome } \\
\text { number }\end{array}$} & \multicolumn{2}{c}{ Relative length $(\mathrm{L} \%)$} & Disomic & \multicolumn{2}{c}{ Arm ratio ( $)+$} \\
\cline { 2 - 4 } 1 & Trisomic & 10.59 & Trisomic & Disomic \\
\hline 2 & 9.93 & 9.94 & $1.00 \mathrm{M}$ & $1.00 \mathrm{M}$ \\
3 & 8.41 & 9.43 & $3.10 \mathrm{SM}$ & $1.20 \mathrm{M}$ \\
4 & 8.41 & 8.78 & $1.40 \mathrm{M}$ & $1.85 \mathrm{SM}$ \\
5 & 8.41 & 8.78 & $2.60 \mathrm{SM}$ & $1.83 \mathrm{SM}$ \\
6 & 8.21 & 8.78 & $1.00 \mathrm{M}$ & $1.83 \mathrm{SM}$ \\
7 & 8.27 & 8.14 & $1.50 \mathrm{M}$ & $1.83 \mathrm{SM}$ \\
8 & 7.69 & 8.14 & $12.00 \mathrm{ACRO}$ & $1.66 \mathrm{SM}$ \\
9 & 7.69 & 8.14 & $1.80 \mathrm{SM}$ & $3.00 \mathrm{SM}$ \\
10 & 7.69 & 7.64 & $1.80 \mathrm{SM}$ & $1.50 \mathrm{M}$ \\
11 & 7.02 & 6.97 & $1.40 \mathrm{M}$ & $1.50 \mathrm{M}$ \\
12 & 6.72 & 6.97 & $1.50 \mathrm{M}$ & $1.33 \mathrm{M}$ \\
\hline
\end{tabular}

+ M, SM, ACRO: Metacentric, submetacentric and acrocentric chromosomes, respectively.

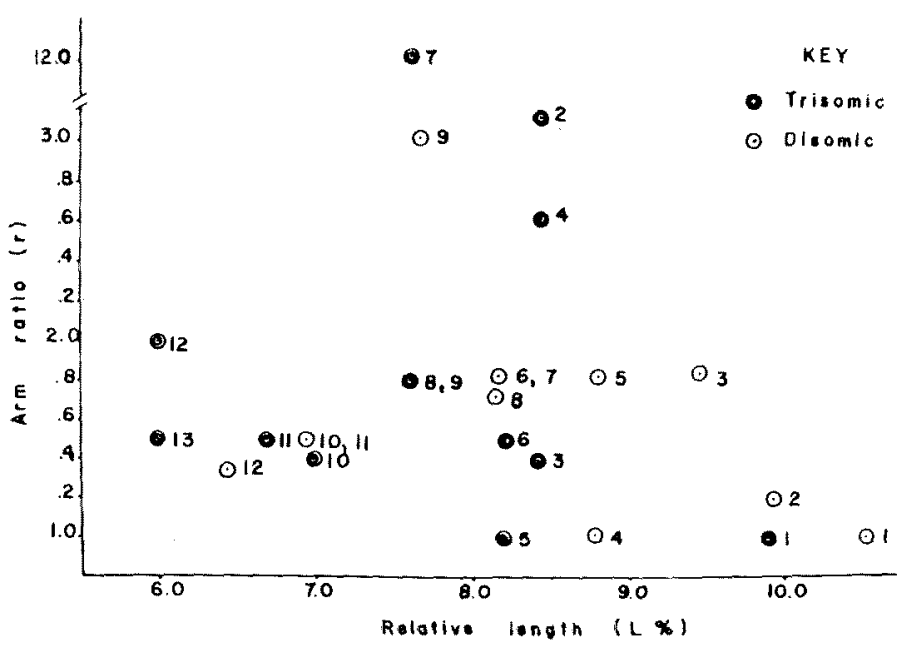

Fig. 2. Graph of relative length (L\%) and arm ratio (r) of chromosome measurements in Table 1. 
A.

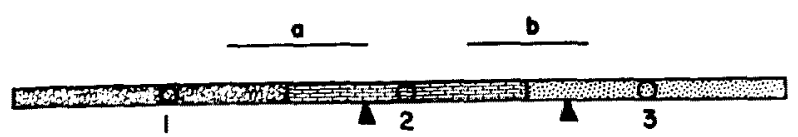

B.

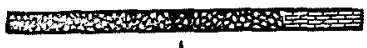

1

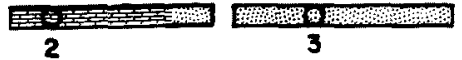

A. Paired chromosomes

$1,2,3$ centromeres

a, b interchangeable chromosome sections

$\Delta$ breakpoint

\section{B. Reorronged chromosomes}

Fig. 3. Schematic origin of translocated chromosomes.

of a mechanism of extensive changes in the complement. Possibly the acrocentric chromosome (number 7 in Table 1, Fig. 2) arose from such unequal interchanges. If one of the largest chromosomes gave rise to the acrocentric, the parent chromosome must have lost one arm. However, since the long arm of the acrocentric is longer than any of the long arms observed in the plant GAVA 1.1, this chromosome may have lost almost one whole arm and gained a segment in the long arm (Fig. 3). Therefore, the hypothesis of the telomere suggested by Muller (1940) and McClintock (1941, 1942) as protecting the ends of chromosome against degradation does not seem to hold in Rhoeo. Discovery of permanent hybridity has been found in Gibasis pulchella in which interchanges of unequal size are confined to terminal segments (Kenton et al. 1987).

They are able to pair end-to-end (Fig. 3A, 3B) with one another and take an unequal interchange of chromosome segments, as the acrocentric chromosome of the plant GAVA 1.1.15. In yeast it has been observed that healing of broken chromosome ends could also involve recombination (Dunn et al. 1984). This agrees with findings in the complex heterozygote of Rhoeo, where pachytene synapsis involves only a small terminal segment (Stack and Soulliere 1984).

It has been observed that in barley, the broken ends of chromosomes became spontaneously healed (Swanson 1957). Acrocentric chromosomes in barley have been extensively used in genetic analysis (Tsuchiya 1979, 1983, Tsuchiya and Fujigaki 1981, Tsuchiya et al. 1984). In sugar beet Romagosa et al. (1985), supposed that an acrocentric chromosome originated from chromosome 9, which healed the broken end after losing its short arm.

Origin of the acrotrisomic plant

From seeds developed by selfing a desynaptic-SDR mutant (GAVA 1.1) of Rhoeo spathacea, 120 seedlings were obtained. Therefore, from these results it is possible to assume that sperm cells with $6,6+$ Acro, 12 and 13 chromosomes are able to function on diploid stigmas and fuse with both reduced or unreduced eggs. Desai (1965), and Lin et al. (1984) found triploid Rhoeo apparently from spontaneous origin. The absence of triploids in the present study may be due to the genetical background which could be unbalanced between zygote and endosperm. Walters and Gerstel (1948) obtained individuals with $2 n=22$ and $2 n=$ 26 by selfing an autotetraploid, which support the idea that unreduced gametes are able to function and produce genetically balanced individuals. Or there is a genetic balance in Renner 


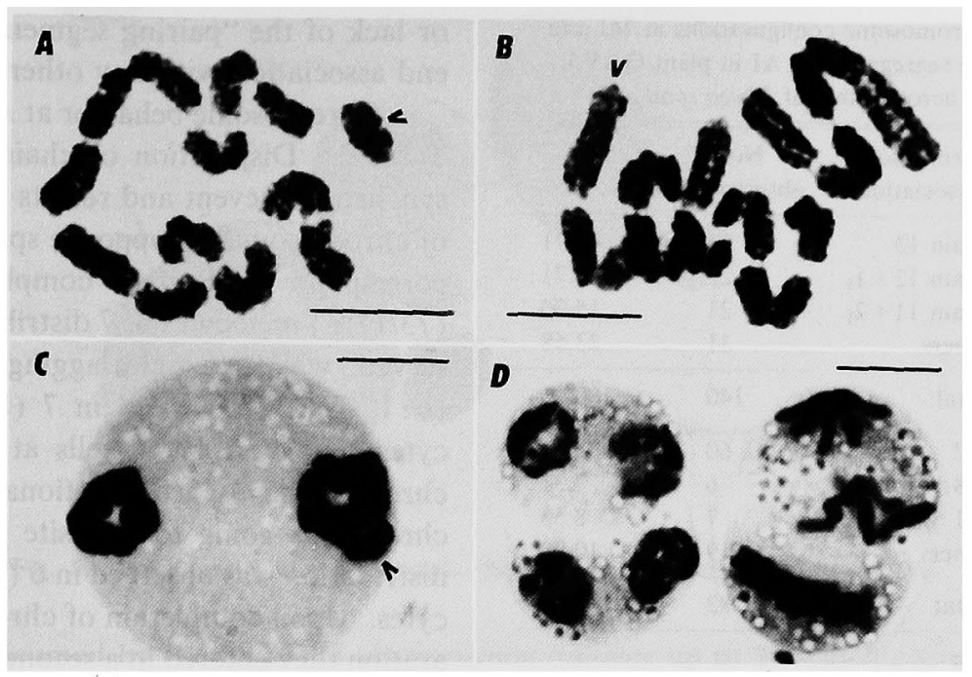

Fig. 4. Meiotic behavior of acrocentric. A. Chain of $9+1_{\mathrm{III}}+1_{\mathrm{I}}$ (acrocentric, arrow) at MI. B. $1_{\mathbf{V I}}+1_{\mathrm{IV}}+1_{\mathrm{II}}+1_{\mathbf{I}}$ (acrocentric, arrow). C. Distribution $6: 7$ at TI, two rings are observed, the extrachromosome is outside of one of them (arrow). D. Tetrad stage but no clear cytokines is observed, asynchrony is observed in both dyad cells with perpendicular orientation. $B a r=10 \mu \mathrm{m}$.

Complexes involved at diploid and tetraploid levels. Apparently the extra chromosome in plants with a somatic chromosome number of $2 n=13$ and $2 n=25$ does not disturb so much that balance. The phenomenon of "triploid block", and the rarity or absence of triploids, in the majority of suitable crosses shows that even when triploids occur, their frequency is far below expectation (Woodel and Valentine 1961).

Within the 120-plants progeny from selfing a supposely homozygous recessive desynaptic, 91 plants were ring-forming (Fig. 4). This is why it is inferred that different types of reproduction took place. Diploid individuals as well as the acrotrisomic were the result of sexual reproduction and recombination ending up as ring formers. Tetraploids and aneutetraploids were asexual and continued to be desynaptics.

The progeny is then a mixture derived from sexual and asexual processes; as is the case in Parthenium (Powers and Rollins 1945). Then it could be said that in Rhoeo apomixis is facultative, having retained some vestige of the sexual cycle from which some recombination potential may be released.

\section{Meiotic behavior}

The meiotic behavior of the acrotrisomic resulted in several unexpected features. Since plant GAVA $1.1 .15(2 n=13)$ was produced by selfing plant GAVA 1.1 , supposed homozygous recessive, ds-SDR, it was expected to observe desynapsis and restitution at the second meiotic division. However, no desynapsis was observed in the many meiocytes studied. Instead, at MI $64(45.7 \%)$ meiocytes showed a chain of 13 chromosomes, 22 (15.71\%) meiocytes presented a chain of $12+1_{1}$, and a chain $11+2_{1}$ was observed in $21(15 \%)$ meiocytes. From Figs. $3 \mathrm{~A}$ and $3 \mathrm{~B}$ it is observed the terminal association of chromatids of homologous segments of chromosome. Other associations were observed in 33 (23.58\%) meiocytes (Table 2). The extra chromosome was found either free as an univalent or at one end of the chain (Figs. 4A, 4B). When associated, its long arm was end-to-end with longest chromosome. The acrotrisomic chromosome always had a free short arm. This could be an indication of homology in the "pairing segment" in both long arms. The free short arm probably indicates a lack of homology 
Table 2. Chromosome configurations at MI and chromosome segregation at AI in plant GAVA

1.1 .15 , an acrotrisomic of Rhoeo spathacea

\begin{tabular}{clcc}
\hline $\begin{array}{c}\text { Meiotic } \\
\text { stage }\end{array}$ & $\begin{array}{c}\text { Chromosome } \\
\text { association }\end{array}$ & $\begin{array}{c}\text { No. of } \\
\text { observed cells }\end{array}$ & $\%$ \\
\hline MI & Chain 13 & 64 & 45.71 \\
& Chain 12+1, & 22 & 15.71 \\
& Chain 11+2, & 21 & 15.00 \\
& Others & 31 & 23.58 \\
\hline \multirow{4}{*}{ AI } & Total & 140 & 100.00 \\
& $6: 7$ & 60 & 73.17 \\
& $5: 8$ & 6 & 7.31 \\
& $6: 1: 6$ & 7 & 8.53 \\
& Others & 9 & 10.99 \\
\hline & Total & 82 & 100.00 \\
\hline
\end{tabular}

or lack of the "pairing segment" for end-toend association with any other arm.

Chromosome behavior at $\mathrm{AI}$ is shown in Table 2. Disjunction of chains is almost a synchronous event and results in two groups of chromosomes at opposite spindle poles, as correspond to Renner complexes. In 60 (73.17\%) meiocytes $6: 7$ distribution was observed, while a single lagging chromosome $(6: 1: 6)$ was observed in $7(8.53 \%)$ meiocytes. In one of the cells at AI the extra chromosome divided equationally with sister chromatids going to opposite poles. A $5: 8$ distribution was observed in $6(7.31 \%)$ meiocytes. Upon completion of chromosome separation they entered in a second formation of rings at both spindle poles (Fig. 4C) with little change in coiling. The many cells observed with such an association in this and another sib plants permit to assume that there is neither randomly aggregation of chromosomes nor an artifact of the technique. The extra chromosome (in 6:7 distribution, at least) is close to the ring but on the outside. Before entering the second meiotic division the second microsporocyte nuclei became diffuse during interkinesis and cytokinesis produce dyads.

This chromosome behavior in the acrotrisomic opens two questions. First: why does the progeny of desynaptic plant reverts to ring forming, when it is supposedly in the homozygous recessive condition after selfing? In most of the synaptic mutants investigated monogenetic recessive inheritance was observed (see for a review, Koduro and Rao 1981). Second: why does such nonhomologous pairing occurs at $\mathrm{TI}$ as it seems to be a ring at both poles? Several cells at TI has been observed with such an association, and they permit to assume that there is not a randomly orientation of such chromosomes. These questions are raised by the unexpected chromosome behavior, but their diplandrogynous autotetraploidy $(2 n=24$ and $2 n=25)$ sibs present a desynaptic behavior (Garcia, unpublished observations).

One possible answer to the first question could be as follows. If the end-to-end pairing is occurring and chromosomes interchange unequal segments, the desynaptic gene effect could be altered by position effect. According with Creighton and McClintock (1931) pairing chromosomes have been shown to exchange parts at the time they exchange genes located on such regions. The observed rings or chains must result from a change in the desynaptic gene. For the other questions there is no answer yet.

Several other alterations at second meiotic division occurred. Metaphase II centromere showed autoorientation and division into sister chromatids at anaphase II. But at this stage alterations in orientation occurred (Fig. 4D), and asynchrony in both cells of the dyad is observed. Also no cleavage furrow was developed in many cells.

\section{Pollen cytology}

Unexpected pollen sizes were obseved (Fig. 5) including several binucleate pollen grains ("siamese twins"); two of which had 26 chromosomes. Since plant GAVA 1.1.15 developed from selfing a ds-SDR (GAVA 1.1, Garcia 1991) unreduced numbers of chromosome was expected. In 58 pollen grains observed, the chromosome number ranged from $n=5$ to $n=26$. Twenty pollen grains presented $n=7(34.4 \%)$ and $13(22.4 \%)$ showed $n=13$ (Fig. 5). Apparently in this acrotrisomic there can be failures in cytokinesis in postmeiotic divisions. 


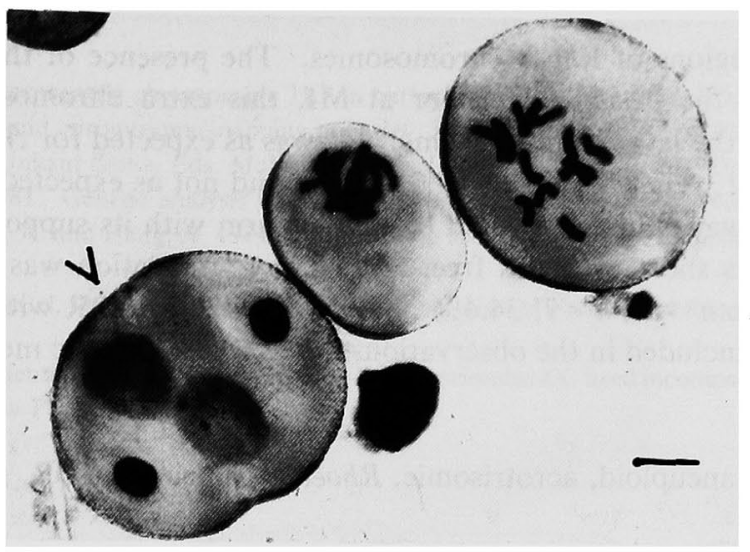

Fig. 5. Pollen grains of various sizes. 'Siasmese twins" (arrow). Bar $=10 \mu \mathrm{m}$.

This can be assumed by the observed chromosome number up to 26 which correspond to the total number of chromatids present in the acrotrisomic, $2 n=13$. From chromosomal segregation observed at AI, pollen grains with $n=6$ and $n=7$ could be expected to occur in high frequencies. Also, if there is a restitution in second division, unreduced pollen grains with chromosome numbers of $n=12$ or $n=14$ are expected. However, pollen grains with $n=13$ which occur in high frequency, and "siamese twins" and pollen grains with $2 n=26$ could have resulted from defective cell wall formation at the sporad stage, resulting in formation of a dyad that is composed of two microspores each containing two daughter nuclei. Matsubayashi (1981) observed that defective cell wall formation is one of the mechanisms of $2 n$ pollen formation in tuber-bearing Solanum species.

The odd number of chromosomes $(n=13)$ supports the idea that not only restitution at second division, of unreduced pollen production is present in Rhoeo. Nuclei produced in the first meiotic division and resulted with 6 or 7 chromosomes will become unreduced with $n=12$ or $n=14$, and then including sister chromatids in a pollen grain. Therefore SDR occurs. However when nuclei with $n=13$ are formed there is an indication that at second meiotic division two nuclei, one with 7 and other with 6 were fused due to aberrant cytokinesis by failure of equational cell wall formation. The high frequency of pollen grains with $n=13$ $(22.4 \%)$ supports this idea and suggests a genetic control of such mechanism. In hybrids of maize $\times$ Tripsacum some disturbances in pollen development were observed, apparently because of failure of chromosomes to congregate at metaphase I plate, tripolar spindle, and failure of cytokinesis at both meiotic divisions (Kindiger 1993). Features of abnormal microspore development were maturation of large pollen grains, "siamese" pollen grain (Kindiger 1993).

In diploid alfalfa (Medicago sativa L.) McCoy and Smith (1983) identified plants which produce "Jumbo" pollen. Jumbo pollen is produced by failure of postmeiotic cytokinesis resulting in four-nucleated pollen. Albertsen and Palmer (1979) refer to these structures in soybeans (Glycine $\max$ L.) as coenocytic microspores. However in the "siamese twins" the four nuclei do not fuse before the first microspore mitosis as is the case in "Jumbo" pollen.

\section{Summary}

The objective of this study in Rhoeo spathacea was the cytological identification of a trisomic plant which includes an acrocentric chromosome. The homology of the extra chromosome was not identified with certainty by karyotype analysis because of its lack of similarity with any other chromosome. Somatic metaphase analysis indicates that interchanges 
take place in terminal regions of $R$ hoeo chromosomes. The presence of the acrocentric also supports this idea. On the basis of behavior at MI, this extra chromosome seems to be homologous with one of the largest chromosome. MI was as expected for ring forming $R$ hoeo (Chains of $13,45.7 \% ; 12+1 \mathrm{I}, 15.7 \%$; or $11+2 \mathrm{I}, 15 \%$ ) and not as expected for a desynaptic. The extra chromosome was either associated by its long arm with its supposed homologue or free; when associated, its short arm was free. At AI the segregation was mostly 6:7, with microspores with $n=5$ to $n=26, n=7,34.4 \%$ and also producing SDR with $n=13(22.4 \%)$. When all the progeny is included in the observations, a possible apomictic mechanism of origin was postulated.

Key words: segmental aneuploid, acrotrisomic, Rhoeo, apomeiotic, SDR.

\section{References}

Albersten, M. C. and Palmer, R. G. 1979. A comparative light and electron-microscopy study of microsporogenesis in male sterile $\left(\mathrm{ms}_{1}\right)$ and male fertile soybeans (Glycine $\max \mathrm{L}$.) Merr. Am. J. Bot. 66: 253-265.

Cleland, R. E. 1972. Oenothera: Cytogenetics and Evolution. Academic Press: New York.

Creighton, B. R. and McClintock, B. 1931. A correlation of cytological and genetical crossing-over in Zea mays. Proc. Natl. Acad. Sci. 17(8): 492-497.

Darlington, C. D. 1929. Ring-formation in Oenothera and other genera. J. Genetics 20: 345-363.

Desai, S. 1965. A cytological study of a triploid Rhoeo discolor. Cytologia 30: 260-265.

Dunn, B., Azauter, P., Pardue, M. L. and Azostak, J. 1984. Transfer of yeast telomeres to linear plasmids by recombination. Cell. 39: 191-201.

García, V. A. 1991. Cytogenetical studies in Rhoeo spathacea (Commelinaceae). I. A desynaptic and second division restitution mutant. Genome 34: 895-899.

- 1994. A desynaptic mutant in Rhoeo spathacea (Commelinaceae). Cytologia 59: 399-404.

Goldblatt, P. 1880. Uneven diploid chromosome numbers and complex heterozygosity in Homeria (Tridadeae). Syst. Bot. 5: $337-340$.

Kenton, A., Davies, A. and Jones, K. 1987. Identification of Renner complex and duplication in permanent hybrids of Gibasis pulchella (Commelinaceae). Chromosoma 95: 424-434.

Kindiger, B. 1993. Aberrant microspore development in hybrids of maize $\times$ Tripsacum dactyloides. Genome 36: $987-$ 997.

Koduro, P. R. K. and Rao, M. K. 1981. Cytogenetics of synaptic mutants in higher plants. Theor. Appl. Genet. 59: 197-214.

Lin, Y. J. and Paddock, E. F. 1973. Ring-position and frequency of adjacent distribution of meiotic chromosomes in Rhoeo spathacea. Am. J. Bot. 60: 685-690.

-, - and King, E. L. 1984. Meiotic chromosome configuration in a triploid Rhoeo. Genetica 63: 113-119.

Matsubayashi, M. 1981. Cytological mechanism for the formation of $2 n$ pollen grains in tuberous Solanums. Potato Sci. 1: $29-34$.

McClintock, B. 1941. The stability of broken ends of chromosome in Zea mays. Genetics 26: $234-282$.

- 1942. The fusion of broken ends of chromosomes following nuclear fusion. Proc. Natl. Acad. Sci. U.S.A. 28: 458463.

McCoy, T. J. and Smith, L. Y. 1983. Genetics, cytology and crossing behavior of a alfalfa (Medicago sativa) mutat. resulting in failure of the postmeiotic cytokinesis. Can. J. Gent. Cytol. 25: 390-397.

Muller, H. J. 1940. An analysis of the process of structural changes in chromosomes of Drosophila melanogaster. J. Genet. 40: 1-66.

Natarajan, A. T. and Natarajan, S. 1972. The heterochromatin of Rhoea discolor. Hereditas 72: 323-330.

Powers, L. and Rollins, R. C. 1945. Reproduction and pollination studies on guayule, Parthenium argentatum Gray and $P$. incanum. J. Am. Soc. Agrom. 37: 96-112.

Romagosa, L., Cistue, L., Tsuchiya, T. and Lasa, J. M. 1985. Cytological identification of acrotrisomy in sugar beet. J. Hered. 76: $227-228$.

Stack, S. M. and Soulliere, D. L. 1984. The relation between synapsis and chiasma formation. Chromosoma 90: $72-$ 83.

Swanson, C. P. 1957. Cytology and Cytogenetics. Prentice Hall. Inc. pp. 350-361.

Tjio, L. H. and Hagberg, A. 1951. Cytological studies on some X-ray mutans of barley. An. Estac. Exp. Aula Dei 2: 
$149-167$.

Tsuchiya, T. 1979. Acrocentric chromosome $1 \mathrm{~L}^{\mathrm{ls}}$ in barley. Barley Genet. Newsl. 9: 101 -102 .

- 1983. Aneuploidy and chromosome mapping in barley. In Cytogenetics of Crop Plants. M. S. Swaminathan, P. K. Gupta and Unkant Sinha. Eds. McMillan, India Ltd. New Delhi. p. 251-281.

- and Fujigaki, J. 1981. Genetic analysis with acrotrisomic 1L ${ }^{\text {ls }}$. Barley Genet. Newsl. 11: 59-60.

-, Singh, R. J., Shala, A. and Hang, A. 1984. Acrotrisomic analysis in linkage mapping in Barley. Theor. Appl. Gen. 68: $433-439$.

Walters, M. S. and Gerstel, D. V. 1948. A cytological investigation of a tetraploid Rhoeo discolor. Am. J. Bot. 35: 141150 .

Woodell, S. R. and Valentine, D. H. 1961. Studies in British primulas IX. Seed incompatibility in diploid-autotetraploid crosses. New Phytol. 60: 282-294. 